\title{
Bourgne, P., Drevet, C., Fourt, X. et Gay-Charpin, M.H. (dir.), Matérialiser l'Utopie
}

\section{Patrice de La Broise}

\section{(2) OpenEdition}

\section{Journals}

Édition électronique

URL : https://journals.openedition.org/edc/12099

DOI : $10.4000 /$ edc. 12099

ISSN : 2101-0366

Éditeur

Université de Lille

Édition imprimée

Date de publication : 23 juillet 2021

Pagination : 187-190

ISBN : 978-2-917562-25-3

ISSN : $1270-6841$

Référence électronique

Patrice de La Broise, «Bourgne, P., Drevet, C., Fourt, X. et Gay-Charpin, M.H. (dir.), Matérialiser I'Utopie »,

Études de communication [En ligne], 56 | 2021, mis en ligne le 23 juillet 2021, consulté le 04 janvier 2023. URL : http://journals.openedition.org/edc/12099 ; DOI : https://doi.org/10.4000/edc.12099

Ce document a été généré automatiquement le 4 janvier 2023.

Tous droits réservés 


\title{
Bourgne, P., Drevet, C., Fourt, X. et Gay-Charpin, M.H. (dir.), Matérialiser l'Utopie
}

\author{
Patrice de La Broise
}

\section{RÉFÉRENCE}

Bourgne, P., Drevet, C., Fourt, X. et Gay-Charpin, M.H. (dir.) (2020), Matérialiser l'Utopie. Presses universitaires Blaise Pascal.

\section{Patrick Bourgne, Xavier Fourt, Christian Drevet et Marie Hélène Gay-Charpin (dir.), Matérialiser I'Utopie'}

1 "Matérialiser l'Utopie ». Tel est le titre de l'ouvrage collectif co-dirigé par Patrick Bourgne, Xavier Fourt, Christian Drevet et Marie Hélène Gay-Charpin. Chercheurs, architectes et artiste réunissent autour d'eux des auteurs aux sensibilités multiples qui, dans une polyphonie interdisciplinaire, font néanmoins chorus. Tous nous invitent à répondre à une question centrale : « comment procède-t-on quand on veut opérer une rupture dans l'existant, activer des puissances imaginaires ou symboliques qui redonnent du sens au réel, ici et maintenant, en matérialisant les effets de cette rupture?» (p. 261).

2 Ce livre est donc une invitation et procède lui-même, dans sa matérialité, d'une utopie éditoriale. Le contrat de lecture en est ainsi revisité dans un préambule enrichi d'un guide traçant et balisant de multiples parcours possibles.

3 Une lecture linéaire, par livrets thématiques successifs, nous délivre trois clefs ouvrant respectivement trois portes. Les «médiums de l'utopie » forment un premier livret où la problématique du partage donne à lire la réalisation d'un idéal animaliste, vulgarisé par les réseaux socio-numériques et intégré par le marché (Marianne Celka), 
l'expérimentation radiophonique d'une utopie solidaire (Éric Dacheux et Florine Garlot), la traduction d'une innovation urbaine (Lydie Lenne). D'où il ressort que les rêves sociaux renvoient à une "utopie positive ", une espérance génératrice d'images que Patrick Bourgne distingue d'une «utopie négative » en lutte contre l'idéologie et ses images réifiées. Par les voies idéal-typiques d'une matérialisation, le co-directeur de l'ouvrage dénonce des "utopies fantômes" nourries par l'idolâtrie (voie 1), mais observe une "sédimentation des utopies dans un imaginaire qui permet aujourd'hui à des collectifs fondés au départ sur l'activisme politique (voie 5) de trouver un cadre pour organiser la collectivité de façon pérenne (voie 4)».

Dans le livret 2, titré « Terres, sources d'utopie », l'utopie se déprend de l'idéal, du rêve social et des espérances. Sophie Gosselin et David gé Bartoli y défendent l'hypothèse que « la suspension du pouvoir d'État n'aboutit pas nécessairement à la loi du plus fort, ni à l'instauration de communautés identitaires et fermées ». Et de conclure que «le monde n'est possible qu'à devenir terrestre ", que l'imagination est immanente aux corps et que la puissance utopique s'éprouve - à l'instar de la ZAD de Notre-Dame-desLandes - dans la résistance des corps à la fiction arbitraire de l'État. Plus loin, Anders Fjeld nous invite à entendre la "parole muette " du familistère de Guise, celle où l'expérimentation utopique vise à émanciper les travailleurs, mais intègre sans mot dire l'imaginaire capitaliste que viennent consacrer l'organisation du travail, l'efficacité productive et l'idée de "devoir ». Une autre "parole muette » est aussi à entendre dans la contribution de Nathalie Blanc pour qui la maladie peut être envisagée comme utopie du non-dit, "une utopie du corps qui se rend visible, une utopie associée à la matérialité des chairs et des dysfonctionnements socio-naturels, une utopie ouvrant sur une négociation avec les corps propres dans l'optique d'en interpréter les signes » (p. 147).

5 Xavier Four, quant à lui, n'envisage l'utopie que dans «l'expérience d'une réalité qui n'existe pas », laquelle ne relèverait ni de la fiction, ni de la modélisation. L'expérience "concrète, ouverte, sans attente et désenchantée " d'une utopie radicale où la communauté utopique s'éprouverait dans l'abolition des lois et l'expérience de l'inappropriabilité (c'est-à-dire la destitution de la propriété).

Cette radicalité referme le second livret avant d'inaugurer celui dédié à « l'ambivalence de l'utopie " que trois contributions viennent discuter. La première, relative à la "communauté intentionnelle» d'Auroville située en Inde du Sud, nous montre combien des espaces de créativités sociales peuvent ouvrir « les humains à de nouvelles perspectives, à de nouveaux paradigmes et à de nouvelle manières de faire » (p. 189). $\mathrm{Au}$ terme d'une étude longue de six ans, Marie Horasius ne propose pas la vision idyllique d'une société indifférente à toute contingence : la dynamique sociale, toujours en recherche d'alternatives y reste guidée par des valeurs fondamentalement humanistes qui font simultanément d'Auroville une «utopie pour touristes » et une " société en mouvement pour ses habitants ». Ici réside l'ambivalence d'un projet vieux de cinquante ans, où « faire société » oblige au pragmatisme.

7 Cette étude de cas trouve un écho dans la proposition théorique d'Anaïs Bovet (chapitre 10) qui, questionnant l'imaginaire utopique, défend que cet imaginaire ne puisse se détacher de la réalité. L'auteure reprend ici la proposition de Mannheim² visant à définir l'utopie à partir de l'action. Ainsi s'ouvre «(...) la perspective d'une matérialité utopique qui émanerait des acteurs aspirant à la transformation, matérialité qui serait celle de leurs actes en vue de la transformation souhaitée » 
(p. 202). Horizon inatteignable, l'utopie guide néanmoins la caravane, «matérialité mouvante » qui l'incarne.

8 L'architecte Christian Drevet ne dit pas autre chose quand il témoigne des influences, non directement constructives, que les utopies ont néanmoins exercé sur l'architecture contemporaine. Revenant à Utopia, l'île artificielle aux cinquante-quatre cités identiques de Thomas More, s'ensuivent et s'entremêlent «l'architecture parlante » d'Étienne-Louis Boullée au Siècle des lumières, le suprématisme architectonique de Kazimir Malevitch, la verticalité de Iannis Xenakis, la ville spatiale de Yona Friedman, l'utopie concrète de Roland Castro... S'il ne fait pas de doute que l'utopie trouve dans l'architecture une matérialisation remarquable, la rétrospective est cependant trompeuse en ce que la fabrication de l'architecture contemporaine n'opère pas dans un temps chronologique ou historique, mais plus sûrement anachronique. Et Christian Drevet de s'interroger: "Quelles matérialisations ont apportées 500 ans d'utopie à l'état actuel de l'architecture ? (...) La réponse réside précisément dans l'entrelacement instable et ambivalent de notions, de valeurs et de questions qui constitue la base génétique de notre espace architectural » (p. 234).

$9 \mathrm{Au}$ demeurant, cet entrelacement vaut aussi pour les contributions de l'ouvrage présenté ici, lequel, outre la trilogie de ses livrets, peut être lu selon d'autres associations thématiques proposées en préambule, comme aussi au fil des illustrations mettant en dialogue l'artiste et l'écrivain, et d'autres choix iconographiques montrant le caractère sensible de l'utopie.

Oui, cet ouvrage procède d'une utopie éditoriale en ce qu'il n'est pas un «mode d'emploi de la matérialisation des utopies", mais se risque à un travail de collecte d'expériences éprouvant l'opérativité des analyses et des concepts. Certaines contributions, à l'instar du texte " hors champ » de Roger Winterhalter, échappent aux codes et références académiques. L'auteur y livre l'expérience d'une " utopie en actes " dans un petit village alsacien, Lutterbach. Une expérience vécue de la citoyenneté, de la solidarité, du "vivre ensemble ", où l'innovation sociale ne rechigne pas à la poésie et au rêve. Ce récit impressionniste n'est cependant pas incongru dans un ouvrage collectif où s'entremêlent les contributions spéculatives et érudites, mais toujours instructives. Certes, le lecteur pourra se perdre dans les méandres d'un ensemble protéiforme où les modes de matérialisation de l'utopie résistent tout autant à leur enfermement théorique et conceptuel qu'à leur accomplissement. Mais cet ouvrage, dans sa forme même et les expériences qu'il recèle, donne beaucoup à réfléchir sur un objet réputé métaphysique dont les manifestations concrètes, par leur analyse rigoureuse, sont éminemment heuristiques.

Patrice de La Broise

Univ. Lille, ULR 4073 - GERiiCO, F-59000 Lille, France

patrice.de-la-broise@univ-lille.fr 


\section{NOTES}

1. Bourgne, P., Drevet, C., Fourt, X. et Gay-Charpin, M.H. (dir.) (2020), Matérialiser l'Utopie. Presses universitaires Blaise Pascal.

2. Mannheim, K. (1929/1956). Idéologie et utopie. Introduction à la sociologie de la connaissance (P. Rollet, Trad.). M. Rivière et Cie.

\section{AUTEURS}

\section{PATRICE DE LA BROISE}

Univ. Lille, ULR 4073 - GERiiCO, F-59000 Lille, France, patrice.de-la-broise@univ-lille.fr 